\title{
A Vision for Neutrino Particle Physics at the South Pole: from IceCube to PINGU
}

\author{
João Pedro Athayde Marcondes de André* for the IceCube-Gen2 collaboration! \\ Michigan State University \\ E-mail: jpandre@msu.edu
}

\begin{abstract}
Located at the South Pole Station in Antarctica, the IceCube Neutrino Observatory is the world's largest neutrino telescope. In the clearest part of the ice sits a more densely instrumented section, DeepCore, that is able to measure neutrinos from $5 \mathrm{GeV}$ to $80 \mathrm{GeV}$. Using DeepCore, neutrino oscillations can be observed via $v_{\mu}$ disappearance with precision comparable to that from accelerator experiments. With additional optical modules instrumenting the DeepCore volume, it is possible to further reduce the detector's energy threshold and improve the resolution of the detector at low energies. This allows measuring the $v_{\tau}$ appearance - which accompanies $v_{\mu}$ disappearance - at $10 \%$ precision or better, and determining the neutrino mass ordering. These are the key science goals of the proposed IceCube-Gen2 Phase 1 and PINGU, respectively.

Both current IceCube results on standard neutrino oscillations and sensitivities for the proposed Phase 1 and PINGU extensions of IceCube will be discussed in this talk.
\end{abstract}

XVII International Workshop on Neutrino Telescopes

13-17 March 2017

Venezia, Italy

* Speaker.

${ }^{\dagger}$ see http://icecube.wisc.edu/collaboration/authors/icecubegen2_2017 for author list 


\section{Introduction}

Neutrino oscillations were discovered by Super-Kamiokande in 1998 [1] through the measurement of atmospheric neutrinos, and by SNO in 2002 [2] through the measurement of solar neutrinos. Since then, neutrino oscillations have repeatedly been observed using many different neutrino sources. As a result, the parameters describing the standard three flavor neutrino oscillations have been determined with increasing precision by many different experiments (see Ref. [3] and references therein) with the exception of the CP-violating phase $\left(\delta_{C P}\right)$ and the mass ordering (the sign of $\Delta m_{32}^{2}$ ). In the case of standard neutrino oscillations, their amplitude is determined by the elements of the 'PMNS' mixing matrix, described by the mixing angles $\left(\theta_{12}, \theta_{13}\right.$, and $\left.\theta_{23}\right)$ and $\delta_{C P}$, while their period in vacuum depends on $\left|\Delta m_{32}^{2}\right| L / E$ and $\left|\Delta m_{21}^{2}\right| L / E$, where $E$ is the neutrino energy, $L$ is the distance between its production and detection points, and $\Delta m_{j i}^{2}$ is the difference between the square of the masses of the mass eigenstates $v_{j}$ and $v_{i}$.

Atmospheric neutrinos are particularly interesting for studying neutrino oscillations. This is because they are naturally produced with energies spanning many orders of magnitude and are available for study, with $L$ varying from a few kilometers to the Earth's diameter of about $12700 \mathrm{~km}$. In an atmospheric neutrino detector we reconstruct the neutrino direction, thereby uniquely determining the propagation baseline $L$ for that oscillation, as well as the neutrino energy $E$. With these observables, we then map the neutrino oscillation pattern in the two-dimensional $L \times E$ space.

It is also worth noting that thanks to the extremely long baselines available for atmospheric neutrinos, the energies at which we observe neutrino oscillations is significantly higher than for accelerator-based experiments. This is essential for verifying that we indeed observe the same neutrino oscillations at different energies, which is what we expect with the $L / E$ dependency. In addition we also provide neutrino oscillation measurements where nuclear re-interaction effects are significantly less important than for current accelerator experiments. This is the case because neutrinos with energy around $25 \mathrm{GeV}$, which corresponds to the first maximal muon neutrino disappearance for up-going neutrinos, mainly interact via deep inelastic scattering [4].

The higher energy also opens the possibility to search for tau neutrino appearance, as the high tau mass suppresses the tau neutrino charged current (CC) interaction at low energies. While it has been established that tau neutrinos do appear as the muon neutrinos disappear [5, 6], we have only poor constraints on the $\tau$ row of the neutrino mixing matrix [7] in comparison to the $e$ and $\mu$ rows due to the lack of neutrino oscillation data where $v_{\tau}$ appearance can be observed.

Given the Earth's density profile [8] and the relatively large measured value of $\theta_{13}[9,10,11$, $12,13]$, additional neutrino mass ordering dependent effects appear at around $5 \mathrm{GeV}$ to $15 \mathrm{GeV}$ for core and mantle crossing trajectories $[14,15,16,17,18]$. While these effects have little influence on the measurement of neutrino oscillations at the first maximum, they can be used to determine the neutrino mass ordering if the neutrino direction and energy can be reconstructed with enough precision and the sample sizes are large enough. While a $3 \sigma$ determination of the neutrino mass ordering is not within reach of the current IceCube detector it could be achieved with the proposed IceCube-Gen2 PINGU detector.

In this talk we will discuss recent results on standard neutrino oscillations in the IceCube Neutrino Observatory, and the sensitivity of IceCube-Gen2, a proposed augmentation of the current IceCube detector. 


\section{The IceCube detector}

The IceCube Neutrino Observatory [19] is the world's largest neutrino detector, with a total volume of about $1 \mathrm{~km}^{3}$ in the deep glacier near the South Pole Station, Antarctica, and is instrumented with 5160 digital optical modules (DOMs), as shown in Fig. 1. The observatory was originally designed to detect $\mathrm{TeV}-\mathrm{PeV}$ neutrinos. An astrophysical flux was indeed discovered in this energy range in 2014 [20]. In 2008 the original design was augmented by creating a central region called DeepCore [21] in the deepest, clearest ice with a higher density of DOMs. This increased density of optical modules over 10 Mton of ice reduces the energy threshold of IceCube from hundreds to a few $\mathrm{GeV}$ and makes it possible to perform competitive neutrino oscillation measurements and searches for dark matter signals.

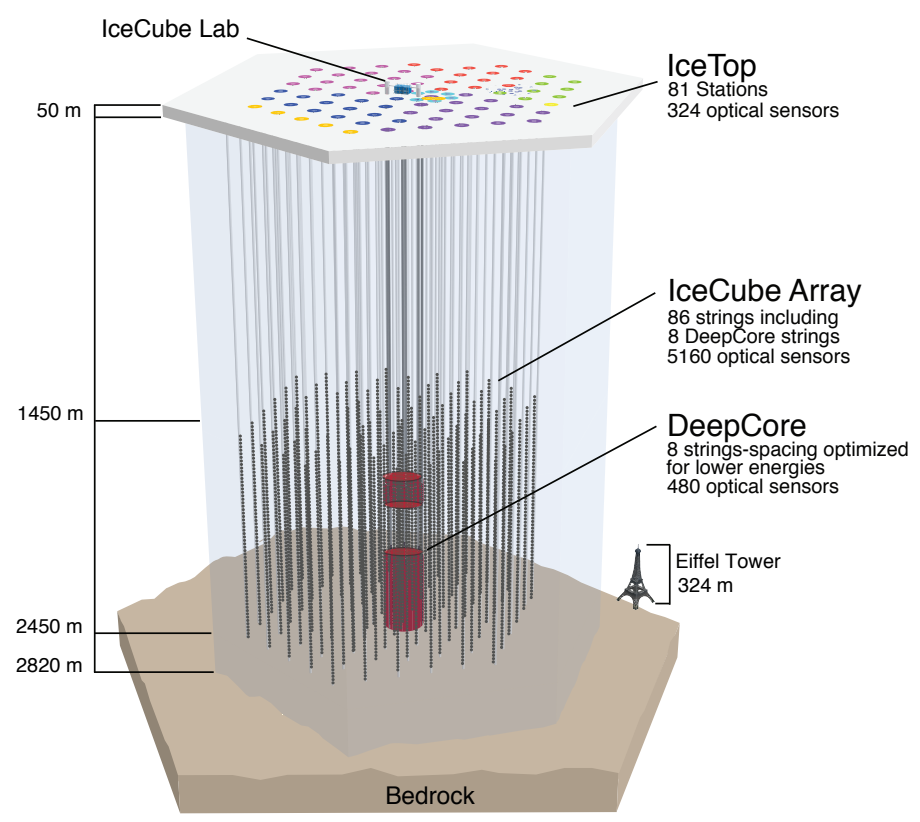

Figure 1: Diagram of the IceCube Neutrino Observatory at its completion, December 2010, with the denser DeepCore array indicated.

IceCube detects neutrinos by measuring the Cherenkov light produced from charged particles created by the neutrinos interacting in the ice or bedrock. The resulting hadronic or electromagnetic showers will emit most of the light close to the interaction vertex, and the observed event is roughly spherical. When a muon is produced in the neutrino interaction it propagates through the ice emitting Cherenkov light over a long distance, and the observed event is more elongated. These two topologies are identified as "cascade-like" and "track-like", respectively.

For the energies relevant for standard neutrino oscillation analyses, the "track-like" sample will be composed mainly of muon neutrino interactions, while the "cascade-like" sample will be more evenly split between misidentified muon neutrinos and electron neutrinos, as shown in Fig. 2, with most of the tau neutrinos and neutral current interactions being classified as "cascade-like". Given the principal oscillation effect observed at these energies is the $v_{\mu} \rightarrow v_{\tau}$ transition, the creation of 


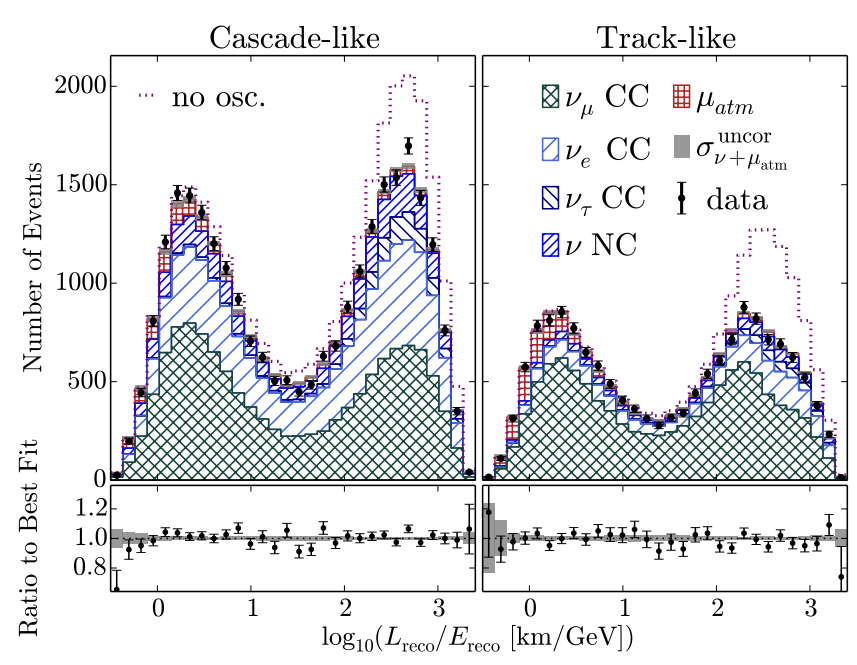

Figure 2: DeepCore $v_{\mu}$ disappearance data projected onto $L / E$ for illustration. The stacked hatched histograms are the predicted counts given the best-fit values of all parameters in the fit for each component. The dotted line shows the expectation in the absence of neutrino oscillations. The bottom plots show the ratio of the data to the fitted prediction.

a $v_{\mu}$ CC enhanced sample is particularly useful, as is shown by the comparison between the best fit and data to the non-oscillated hypothesis shown in Fig. 2.

To measure the atmospheric oscillation parameters, we fit jointly the $E \times \cos \theta_{z}$ distribution, where $\cos \theta_{z}$ is the cosine of the reconstructed neutrino zenith angle that determines $L$, for both the track-like and cascade-like samples, rather than the $L / E$ distribution shown in Fig. 2. This allows simpler discrimination between the effects of the oscillations under study here and our systematic uncertainties. We consider systematic uncertainties on the neutrino flux, both as a function of energy and direction, on the neutrino cross section, on the background rejection efficiency and from detector effects.

The result obtained with three years of detector data, using the approach of Feldman and Cousins [22] to ensure proper coverage, is shown in Fig. 3. Our results are consistent with those obtained by other experiments [12, 23, 24, 25], even though we observe neutrino oscillations at a significantly higher energy and are thus subject to a very different set of systematic uncertainties.

For a more complete discussion of this result, please refer to Ref. [26].

In addition to measuring for the $v_{\mu}$ disappearance produced by the $v_{\mu} \rightarrow v_{\tau}$ transition, DeepCore also makes it possible to search for the appearance of $v_{\tau}$ events in the "cascade-like" sample, which enables performing an inclusive $v_{\tau}$ appearance rate measurement. This measurement is particularly interesting as a probe to the unitarity of the PMNS matrix. This matrix would not be unitary in particular if there were additional families of neutrinos, which is a popular solution to a series of anomalies observed in various neutrino experiments [27]. Therefore, testing the unitarity of the PMNS matrix is a new way of probing the existence of sterile neutrinos. For additional discussion on direct searches for these additional families of neutrinos in IceCube, the reader is referred to Ref. [28]. 


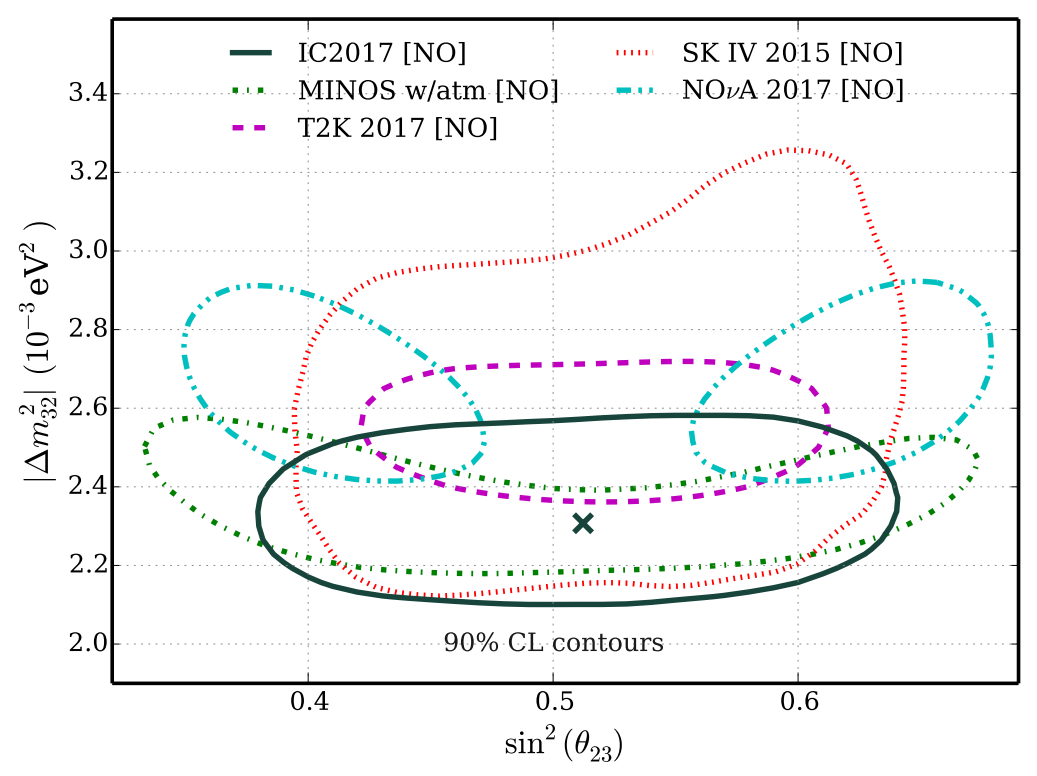

Figure 3: The 90\% CL allowed region for the measurement of the atmospheric neutrino oscillation parameters with IceCube (solid line), with the cross indicating our best fit point assuming normal ordering. For comparison the results obtained by other experiments [12, 23, 24, 25] are also shown (dashed lines).

The afore mentioned study of $v_{\tau}$ appearance is currently underway in IceCube, however no results are available presently. Using the same event selection as for the result discussed above, we expect about $40 \%$ precision on the tau neutrino normalization, which would be comparable or better than the currently available measurements of the tau neutrino appearance rate $[5,6]$.

\section{The IceCube-Gen2 Phase1 upgrade}

A significant enhancement of the $v_{\tau}$ appearance measurement, leading to a $10 \%$ precision on the $v_{\tau}$ normalization, requires improvements to the detector. These will aid in the $v_{\mu}$ CC identification, in order to reduce the fraction of the "cascade-like" sample composed of misidentified $v_{\mu} \mathrm{CC}$ events, and in the reconstruction of those "cascade-like" events.

The IceCube-Gen2 Phase 1 upgrade proposal addresses these goals by augmenting the current detector with seven new detection strings filling in a small fraction of the DeepCore sub-array, as is shown in Fig. 4. Furthermore, these new strings will be instrumented with multi-PMT optical modules (mDOM), with smaller vertical spacing on the string to significantly increase the photon detection efficiency in that region of the detector.

The additional optical modules deployed with the Phase 1 upgrade will contain new calibration devices whose advantages are twofold: not only will they improve the understanding of the Phase 1 detector and related systematics, but the knowledge gained will also serve as motivation to reanalyse all data taken before the deployment of Phase 1 .

The re-analysis of the data is particularly interesting in the context of neutrino astronomy. The reason is that ice systematics are significantly limiting the angular resolution of highest energy 


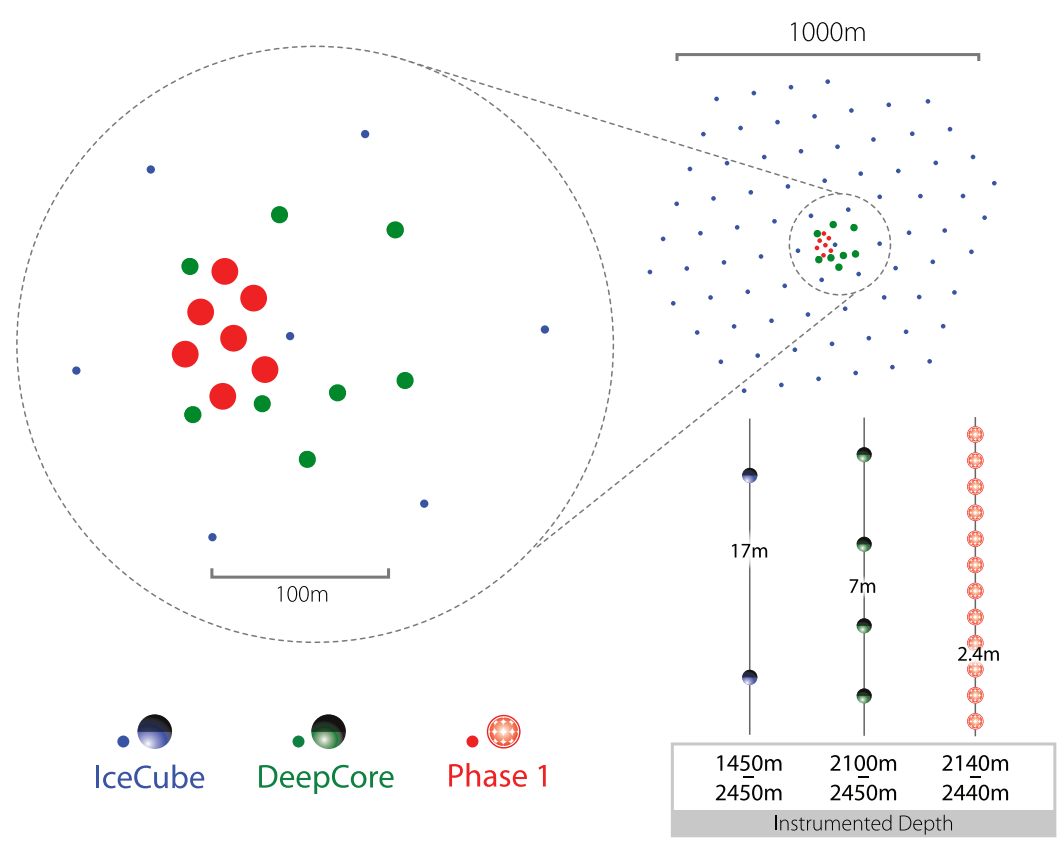

Figure 4: Diagram of the IceCube-Gen2 Phase 1 upgrade in relation to the full IceCube detector. The size of the circles in the zoomed in version indicate the relative photocathode area for optical modules of each string.

"cascade-like" events, which makes looking for corresponding sources in electromagnetic channels significantly more challenging. In the following, however, we focus exclusively on the impact of the Phase 1 upgrade on the neutrino oscillation measurement discussed previously.

Employing the same techniques as in IceCube analysis, we expect a strong enhancement of the precision at which we can determine $\sin ^{2} \theta_{23}$ and $\left|\Delta m_{32}^{2}\right|$, and we expect better than $10 \%$ precision on the measurement of the tau neutrino normalization. The expected sensitivity to both these analyses is shown in Fig. 5.

It is worth highlighting that we expect a $\sim 3 \sigma$ exclusion of maximal mixing and of the second octant of $\theta_{23}$ with IceCube-Gen2 Phase 1 if the true value of $\theta_{23}$ corresponds to the best fit from NOvA [23] in the first octant. Phase 1 should also be able to determine the neutrino mass ordering at the $3 \sigma$ level in 3-8 years, depending on the true value of $\theta_{23}$.

\section{The IceCube-Gen2 PINGU detector}

As discussed previously, matter effects will induce changes in the neutrino oscillation pattern around $5 \mathrm{GeV}$ to $15 \mathrm{GeV}$ due to the Earth density profile. These will mainly impact neutrinos (anti-neutrinos) if the neutrino mass ordering is normal (inverted). While the atmospheric fluxes of neutrinos and anti-neutrinos are of similar magnitude, the difference between the interaction cross-section of neutrinos and anti-neutrinos [4] makes it possible to measure the neutrino mass ordering even without having a detector capable of differentiating neutrinos from anti-neutrinos as proposed by $[30,31]$. This is achieved by comparing the observed neutrino distribution with the expected distributions under the normal (NO) and inverted (IO) orderings as a function of $L \times E$, in 

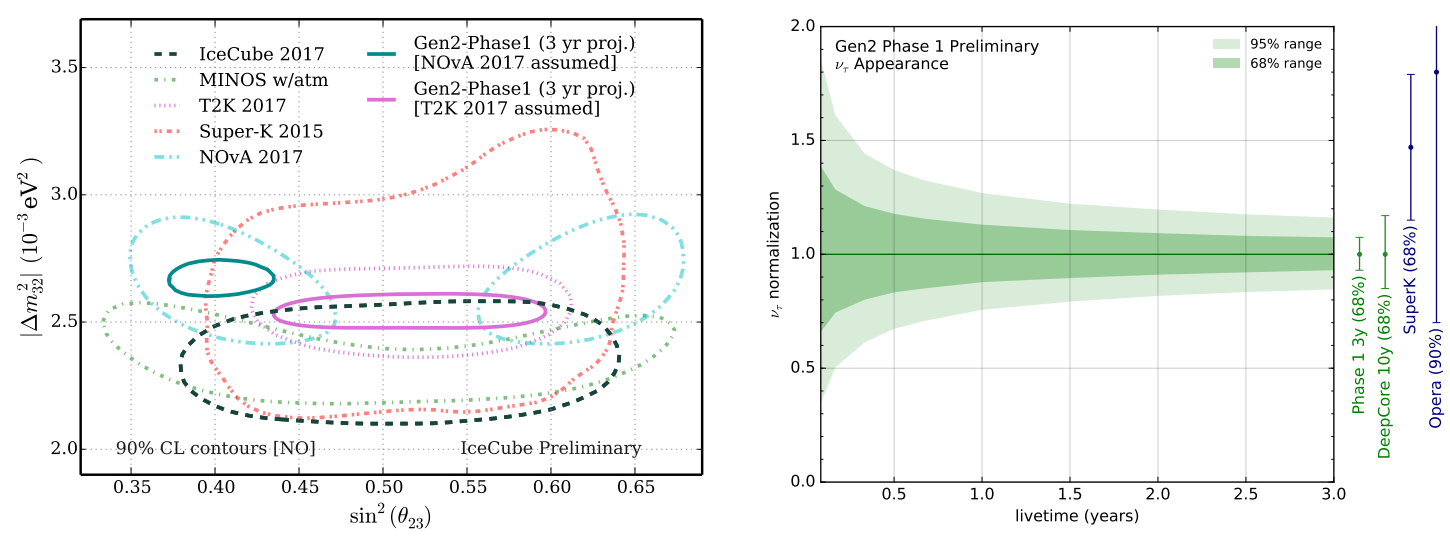

Figure 5: Sensitivity of the Phase 1 upgrade to $\sin ^{2} \theta_{23}$ and $\left|\Delta m_{32}^{2}\right|$ using 3 years of data (left) and to the $v_{\tau}$ normalization as a function of time (right). In both cases for comparison the results obtained by current experiments $([12,23,24,25,26]$ on the left and [5, 29] on the right) are also shown.

analogy to the muon neutrino disappearance analysis discussed earlier in these proceedings. While the expected difference between these two cases is small, the change produces a distinctive pattern in the $L \times E$ distribution, as shown in Fig. 6. It can only be resolved unambiguously with sufficient measurement precision in the key energy range between $5 \mathrm{GeV}$ and $15 \mathrm{GeV}$.
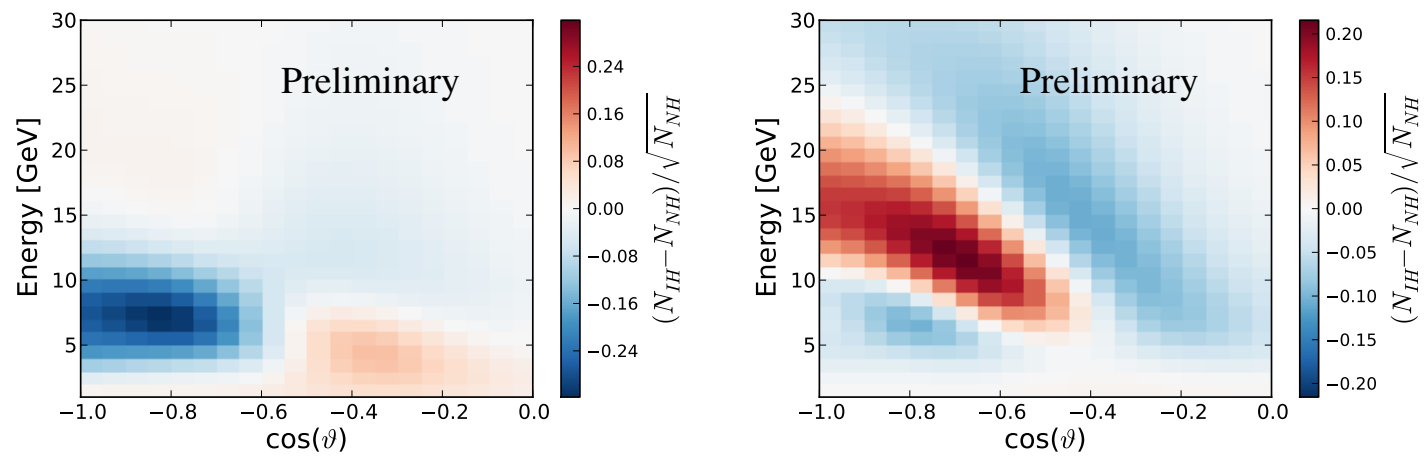

Figure 6: Neutrino mass ordering distinguishability metric as defined in [31] for "cascade-like" (left) and "track-like" (right) events for one year of simulated IceCube-Gen2 PINGU data.

In order to reach $\sim 3 \sigma$ sensitivity for any true $\theta_{23}$ value within 4 years another detector upgrade is required: PINGU. Following the same idea outlined for Phase 1, in PINGU we propose to add 26 strings to the current IceCube detector (that is, 19 strings in addition to the Phase 1 detector), all of those in the DeepCore region. While in all current studies for the PINGU detector we have used optical modules similar to the ones used in IceCube, we plan on changing those to the mDOMs proposed for Phase 1 which should further enhance our sensitivity. The expected median sensitivity to the neutrino mass ordering in IceCube-Gen2 PINGU is shown in Fig. 7.

In addition to contributions to the determination of the neutrino mass ordering highlighted 

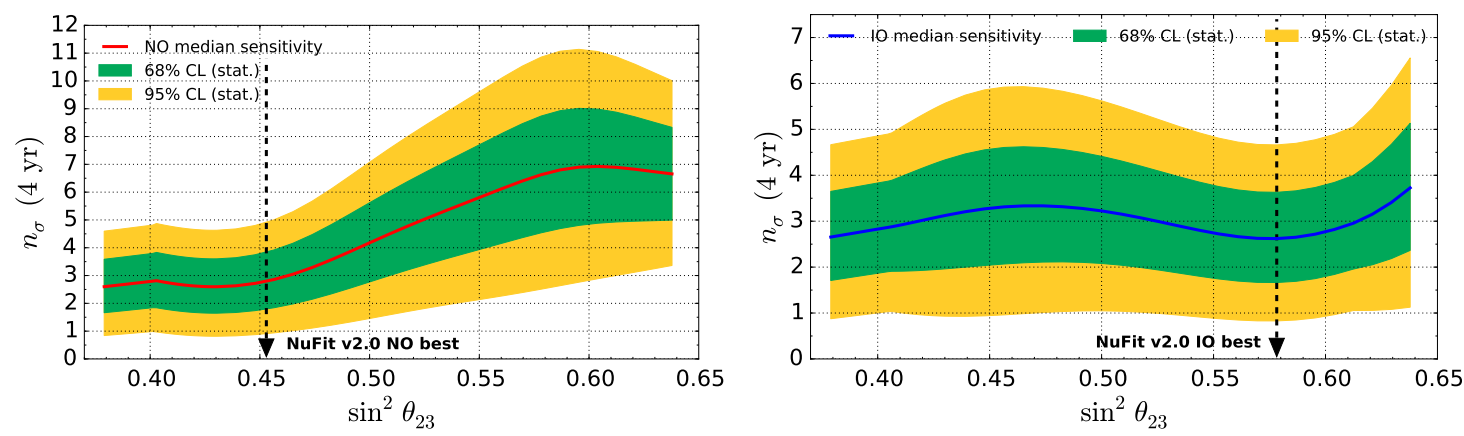

Figure 7: Neutrino mass ordering sensitivity for PINGU assuming normal (left) or inverted (right) ordering as a function of the true $\theta_{23}$ value for 4 years of data. The arrows show the best fit values for $\theta_{23}$ obtained by a recent global fit [32].

above, PINGU will also significantly contribute to the other neutrino oscillation studies discussed here. For additional information on PINGU, please refer to Ref. [33].

\section{Conclusions}

Atmospheric neutrinos have remained a valuable tool to study neutrino oscillations. Using these IceCube has measured the neutrino oscillation parameters with a precision that is competitive to that of accelerator based experiments, but at a significantly higher energy. This makes our results complementary to those from accelerator based experiments in the context of testing the standard neutrino oscillation framework. Additional studies with atmospheric neutrinos in IceCube are still underway to improve the precision of our current results and also aiming towards the first tau neutrino appearance measurement from IceCube.

Going beyond the current detector, proposed extensions to IceCube have a rich physics program. In particular, they will be able to significantly improve IceCube's sensitivity to the atmospheric neutrino oscillation parameters, to tau neutrino appearance searches and to the neutrino mass ordering. With Phase 1, we expect to achieve a precision of better than $10 \%$ on the measurement of the tau neutrino appearance rate, which would greatly facilitate testing the unitarity of the $\tau$ row of the PMNS matrix. PINGU will be able to make a $3 \sigma$ determination of the neutrino mass ordering within 4 years, independent of the true value of $\theta_{23}$.

\section{References}

[1] Y. Fukuda et. al., (Super-Kamiokande) Phys. Rev. Lett. 811562 (1998).

[2] Q. R. Ahmad et. al., (SNO) Phys. Rev. Lett. 89011301 (2002).

[3] K. A. Olive et. al., (Particle Data Group) Chin. Phys. C38 090001 (2014).

[4] J. A. Formaggio and G. P. Zeller Rev. Mod. Phys. 841307 (2012), [1305. 7513 ] .

[5] N. Agafonova et. al., (OPERA) Phys. Rev. Lett. 115121802 (2015), [1507.01417] .

[6] K. Abe et. al., (Super-Kamiokande) Phys. Rev. Lett. 110181802 (2013), [1206.0328 ] . 
[7] S. Parke and M. Ross-Lonergan Phys. Rev. D93 113009 (2016), [1 508.05095 ] .

[8] A. M. Dziewonski and D. L. Anderson Phys. Earth Planet. Interiors 25297 (1981).

[9] F. P. An et. al., (Daya Bay) Phys. Rev. D95 072006 (2017), [ 1610.04802 ] .

[10] S. H. Seo et. al., (RENO) "Spectral Measurement of the Electron Antineutrino Oscillation Amplitude and Frequency using 500 Live Days of RENO Data,” [1610.04326] .

[11] Y. Abe et. al., (Double Chooz) JHEP 01163 (2016), [1510. 08937 ] .

[12] K. Abe et. al., (T2K) Phys. Rev. Lett. 118151801 (2017), [1701.00432 ].

[13] P. Adamson et. al., (NOvA) Phys. Rev. Lett. 118231801 (2017), [ 1703.03328 ] .

[14] L. Wolfenstein Phys. Rev. D17 2369 (1978).

[15] S. P. Mikheev and A. Yu. Smirnov Sov. J. Nucl. Phys. 42913 (1985).

[16] S. T. Petcov Phys. Lett. B434 321 (1998), [hep-ph/9805262 ].

[17] E. K. Akhmedov et. al. JHEP 05077 (2007), [ hep-ph/ 0612285 ] .

[18] E. K. Akhmedov et. al. JHEP 06072 (2008), [ 0804.1466 ] .

[19] M. G. Aartsen et. al., (IceCube) JINST 12 P03012 (2017), [ 1612.05093 ] .

[20] M. G. Aartsen et. al., (IceCube) Phys. Rev. Lett. 113101101 (2014), [1405. 5303 ] .

[21] R. Abbasi et. al., (IceCube) Astropart. Phys. 35615 (2012), [1109.6096] .

[22] G. J. Feldman and R. D. Cousins Phys. Rev. D57 3873 (1998).

[23] P. Adamson et. al., (NOvA) Phys. Rev. Lett. 118151802 (2017), [1701.05891] .

[24] P. Adamson et. al., (MINOS) Phys. Rev. Lett. 110251801 (2013).

[25] R. Wendell et. al., (Super-Kamiokande) AIP Conf. Proc. 1666100001 (2015).

[26] M. G. Aartsen et. al., (IceCube) "Measurement of Atmospheric Neutrino Oscillations at 6-56 GeV with IceCube DeepCore," [1707.07081].

[27] K. N. Abazajian et. al. "Light Sterile Neutrinos: A White Paper," [1204.5379] . And references therein.

[28] A. Terliuk et. al., (IceCube) PoS(NEUTEL2017)045. in these proceedings.

[29] Z. Li, (Super-Kamiokande, Hyper-Kamiokande) Nucl. Part. Phys. Proc. 287-288 147 (2017).

[30] O. Mena, I. Mocioiu, and S. Razzaque Phys. Rev. D78 093003 (2008), [ 0803.3044 ] .

[31] E. K. Akhmedov et. al. JHEP 02082 (2013), [1205. 7071 ] .

[32] M. C. Gonzalez-Garcia, M. Maltoni, and T. Schwetz JHEP 11052 (2014), [ 1409.5439 ] .

[33] M. G. Aartsen et. al., (IceCube) J. Phys. G44 054006 (2017), [1607.02671 ] . 